\title{
Forest inventory sensitivity to UAS-based image processing algorithms
}

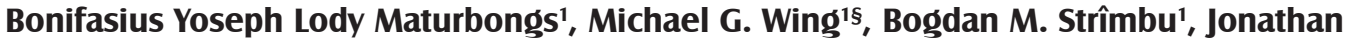 \\ Burnett $^{2}$
}

Maturbongs B.Y.L., Wing M.G., Strîmbu B.M., Burnett J., 2019. Forest inventory sensitivity to UAS-based image processing algorithms. Ann. For. Res. 62(1): 87-108.

Abstract. Frequent and accurate estimation of forest structure parameters, such as number of trees per hectare or total height, are mandatory for sustainable forest management. Unmanned aircraft system (UAS) equipped with inexpensive sensors can be used to monitor and measure forest structure. The detailed information provided by the UAS allows tree level forest inventory. However, tree identification depends on a variety of parameters defining the image processing and tree segmentation algorithms. The objective of our study was to identify parameter combinations that accurately delineated trees and their heights. We evaluated the impact of different tree segmentation and point cloud generation algorithms on forest inventory from imagery collected with a UAS over a mature Douglas-fir plantation forest. We processed the images with two commonly used commercial software packages, Agisoft PhotoScan and Pix4Dmapper, both implementing image processing algorithms called Structure from Motion. For each software we generated photogrammetric point clouds by varying the parameters defining the implementation. We segmented individual trees and heights using three tree algorithms: Variable Window Filter, Graph-Theoretical, and Watershed Segmentation. We assessed the impact of image processing algorithms on forest inventory by comparing the estimated trees with trees manually identified from the point clouds. We found that the type of tree segmentation and image processing algorithms have a significant effect in accurately identifying trees. For tree height estimation, we found strong evidence that image processing algorithms had significant effects, whereas tree segmentation algorithms did not significantly affect tree height estimation. These findings may be of interest to others that are using high-resolution spatial imagery to estimate forest inventory parameters.

Keywords: Unmanned Aircraft Systems (UAS), photogrammetric point clouds, trees segmentation, structure from motion parameters, Douglas-fir

Authors. ${ }^{1}$ College of Forestry, Oregon State University, Corvallis OR USA | ${ }^{2}$ US Forest Service, Pacific Northwest Station, Corvallis OR USA

${ }^{\S}$ Corresponding author: Michael G. Wing (michael.wing@oregonstate.edu)

Manuscript received May 2, 2019; revised July 23, 2019; accepted July 24, 2019; online first July 30, 2019. 


\section{Introduction}

Field measurements required for accurate forest inventory assessments are expensive and time consuming to collect on the ground. Furthermore, the information acquired during stand cruising is rarely used in subsequent inventories. Remote sensing inventories are generally less expensive and provide parameters of the population rather than statistical samples, and are commonly used in following resource assessments. Among vehicles that can capture remotely sensed data,small unmanned aircraft systems (UAS) are relatively inexpensive $(<\$ 2000$ USD) and can be an alternative to field measurementsfor forest structureestimation(Wing et al. 2014, Wallace et al. 2016, Sankey et al. 2017, Torresan et al. 2017). A UAS is defined as the associated of components of an aircraft that is intended to operate with no pilot on board (ICAO 2011). Various sensors, such as Light Detection and Ranging (LiDAR), multispectral, hyperspectral, and visible-light (RGB), sometimes called optical, cameras can be mounted on UAS. The relatively affordable cost of small UAS platforms and growing availability of image processing software makes UAS a competitive and powerful alternative for smaller land areas ( $<500$ ha) when compared to the more expensive airborne or spaceborne data. Furthermore, satellite or manned aircraft data usually have limited spatial resolution (Abdollahnejad et al. 2018) and sometimes temporal resolution compared to the UAS data. Thus, satellite and manned aircraft data may not be effective and efficient for frequent forest structure estimation that requires high spatial and temporal resolution. Although UAS are generally not as efficient for large areasas manned aircraft or spaceborne surveys, they are capable of collecting remotely sensed data from forests with greater spatial and temporal resolution. In addition, open source and commercial flight control applications of UAS continue to improve and make UAS more functional and reliable
(Paneque-Gálvez et al. 2014, Messinger et al. 2016).

Frequent and accurate estimation of forest structure parameters, such as number of trees and tree height is fundamental in sustainable forest management. In addition, these parameters are critical in determining land value and productivity. A growing number of studies have assessed the potential application of aerial photographs in forest inventory (Wallace et al. 2016, Ota et al. 2017), including biomass (Ota et al. 2015, Kachamba et al. 2016) and carbon (Messinger et al. 2016). Optical imagery (RGB) can be defined as imagery data consisting of red, green, and blue wavelengths visible to the human eye. With advancements in computer vision and photogrammetry called Structure from Motion (SFM), aerial photographs now can be processed into high density photogrammetric point clouds (Snavely et al. 2008). SFM can serve as a low-cost alternative to LiDARbyproducing photogrammetric point clouds (PPC). PPC can be generatedwith densities similar to LiDAR (Méndez-Barroso et al. 2018), but at a fraction of the cost with comparable accuracy (Fang \& Strimbu 2017).

Structure from Motion (SFM) implemented from UAS imagery has become more common in many fields including forestry (Mlambo et al. 2017). UAS-based canopy height models (CHM) generated from SFM PPCs can be used to estimate tropical forest biomass (Ota et al. 2015). Messinger et al. (2016) found that UAS-based canopy height and carbon density measurements based on SFM algorithm were strongly correlated with LiDAR measurements. In addition, UAS-based photogrammetric point clouds created with SFM have been used to accurately estimate forest structure (Ota et al. 2017, Panagiotidis et al. 2017, Abdollahnejad et al. 2018) such as Pléiades, it is now possible to estimate tree parameters at the individual level with high fidelity. Despite innovative advantages on high-precision satellites, data acquisition is not yet available to the public at a reasonable cost. Although there are 
studies that have assessed SFM use in UASbased photographs to estimate forest structure, the impact of UAS-based image processing algorithms on forest structure estimation has not been formally explored and assessed, especially from algorithmic perspectives.

We evaluated the impact of different UASbased image processing algorithms and tree segmentation algorithms on forest structure estimation from high resolution imagery collected by a UAS flown over a mature Douglas-fir plantation forest. Our objectives were to examine which combinations led to accurate and reliable tree identification and tree height estimation for forest inventory support. We processed the images with two commonly used commercial software packages, Agisoft PhotoScan and Pix4Dmapper, both implementing image processing algorithms called Structure from Motion (SFM). The parameters within each software package were varied for point cloud generation. For identification of individual trees and their height estimation we used three tree segmentation algorithms: Variable Window Filter (VWF), Graph-Theoretical, and Watershed Segmentation. We assessed the impact of UAS-based image processing algorithms on forest structure estimation by comparing the computed trees with trees manually identified from the 3D point clouds.

\section{Photogrammetric point clouds}

Photogrammetric point clouds PPC generated with SFM algorithms have been found to be similar to airborne LiDAR point clouds (Cook 2017, Méndez-Barroso et al. 2018, Roşca et al. 2018). SFM implements bundle adjustment processes to generate 3D texture of a scene or an object based on its overlapping photographs (Snavely et al. 2008). SFM approaches for high resolution UAS imagery are implemented in some software packages including open source (Mlambo et al. 2017, Singh et al. 2018) and commercial software packages, such as Agisoft PhotoScan (Dandois et al. 2015, Zaha- wi et al. 2015) and Pix4Dmapper (Zhang et al. 2016, Fernández-Guisuraga et al. 2018). Each of these software packages has its own algorithm parameters that define how point clouds are generated.

Agisoft has adjustable algorithm parameters including image alignment, dense point quality, and depth filtering (Agisoft 2018). Image alignment and dense point quality have selectable quality values. Higher quality results in a more detailed and accurate reconstruction of an object but will take longer to process.

Agisoft also has a depth filtering algorithm that will filter outliers as a part of a dense point cloud generation process. In a dense point cloud generation there could be outlier points due to images that are not focused. Wallace et al. (2016) used Agisoft to generate 3D point clouds based on RGB aerial imagery and used high for point cloud quality. Ota et al. $(2015,2017)$ generated point clouds with medium quality using Agisoft, and in another example, Messinger et al. (2016) used Agisoft with highest, ultra-high, and aggressive as the settings for align photos accuracy, dense cloud quality, and depth filtering respectively.

Pix4D has also been used to generate 3D point clouds from UAS imagery (Zhang et al. 2016, Perroy et al. 2017, Carr \& Slyder 2018). Compared to Agisoft, Pix4D has different algorithm parameters including point density, image scale, and minimum number of matches (Pix4Dmapper 2018). The main parameters present in Pix4D are: (i) the point density, which defines the point cloud density, and (ii) the image scale parameter, which controls the image scale in the point cloud generation process, and the minimum number of matches, which defines the minimum number of images that a point should be re-projected in.

\section{Tree segmentation algorithms}

Several tree segmentation algorithms have been widely used in forest inventory, such as the Variable Window Filter (VWF) (Popescu \& 
Wynne 2004), Watershed Segmentation (Goerndt et al. 2010, Edson \& Wing 2011), and the Graph-Theoretical (Strîmbu \& Strîmbu 2015). VWF uses a moving window to define tree tops in a canopy height model (CHM). The size of the moving window depends on the tree height, which assumes that taller trees have wider crowns. Watershed Segmentation delineates individual tree crowns and estimates tree height (Goerndt et al. 2010, Edson \& Wing 2011) by replacing the crown, expressed as a canopy height model, with an inversed basin. The Graph-Theoretical algorithm (Strîmbu \& Strîmbu 2015) is implemented in Tree Extraction (TrEx) software, and identifies individual trees by partitioning an oriented weighted graph according to a set of weights defined by stand structure.

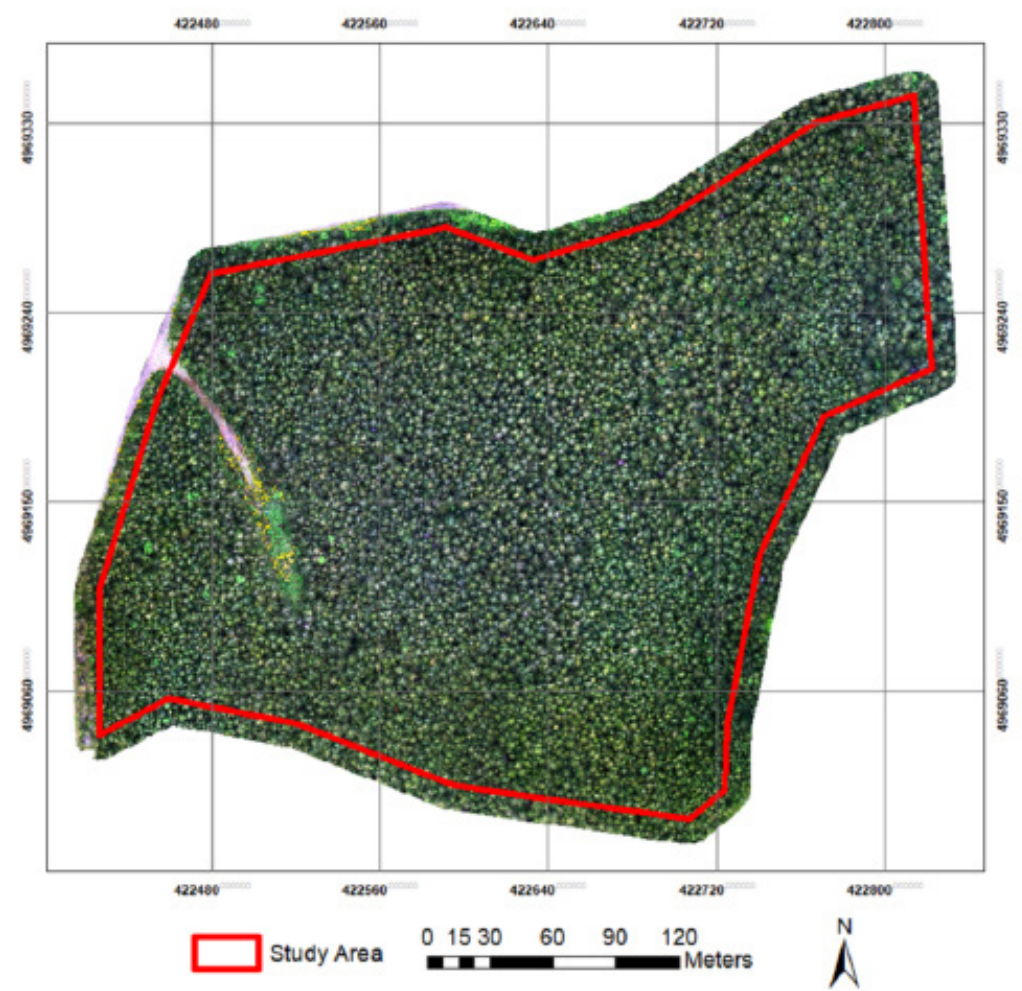

Figure 1 Study area

\section{Materials and methods}

\section{Study area}

The study area was a mature Douglas-fir (Pseudotsuga menziesii) forest plantation on private ownership covering 8.5 ha in the Oregon Coast Range near Lincoln City, Oregon, USA (Latitude $44^{\circ} 52^{\prime} \mathrm{N}$, Longitude $123^{\circ} 58^{\prime}$ W) (Figure 1). Elevation ranged from $159 \mathrm{~m}$ to 243 mabove mean sea level with soils in this area generally being marine sandstone or siltstone. Mean tree diameter and height was $21.8 \mathrm{~cm}$ and $14.0 \mathrm{~m}$, respectively, with a mean crown width of $4.1 \mathrm{~m}$.

Remotely sensed imagery was collected on 26 April 2016 with a Sony A5100 mounted on a Tarot 650 UAS quadcopter. The UAS flew at $120 \mathrm{~m}$ above ground level (AGL) from the takeoff location with 160 images being collected at $80 \%$ overlap and sidelap. The images were in raw format and later converted to high-quality jpegs.

\section{Point clouds generation and normalization}

Our image processing workflow was designed such that comparisons could be made between algorithm outputs and is described in detail below. We produced 3D point clouds from the images using Structure from Motion (SFM) algorithms implemented in Agisoft 
PhotoScan Professional version 1.3.2 (Agisoft 2018) and Pix4Dmapper Pro version 4.2.26 (Pix4Dmapper 2018). Each of these products has two sets of adjustable parameters that we manipulated in the creation of point clouds. Agisoft parameters were image alignment (i.e., high and medium) and dense point quality (i.e., high and medium). Pix4D parameters were image scale (i.e., original and half) and point density (i.e., optimal and low). In addition, there were fixed parameters used in point cloud generation. Fixed parameters for Agisoft were "unchecked" (meaning that this param-

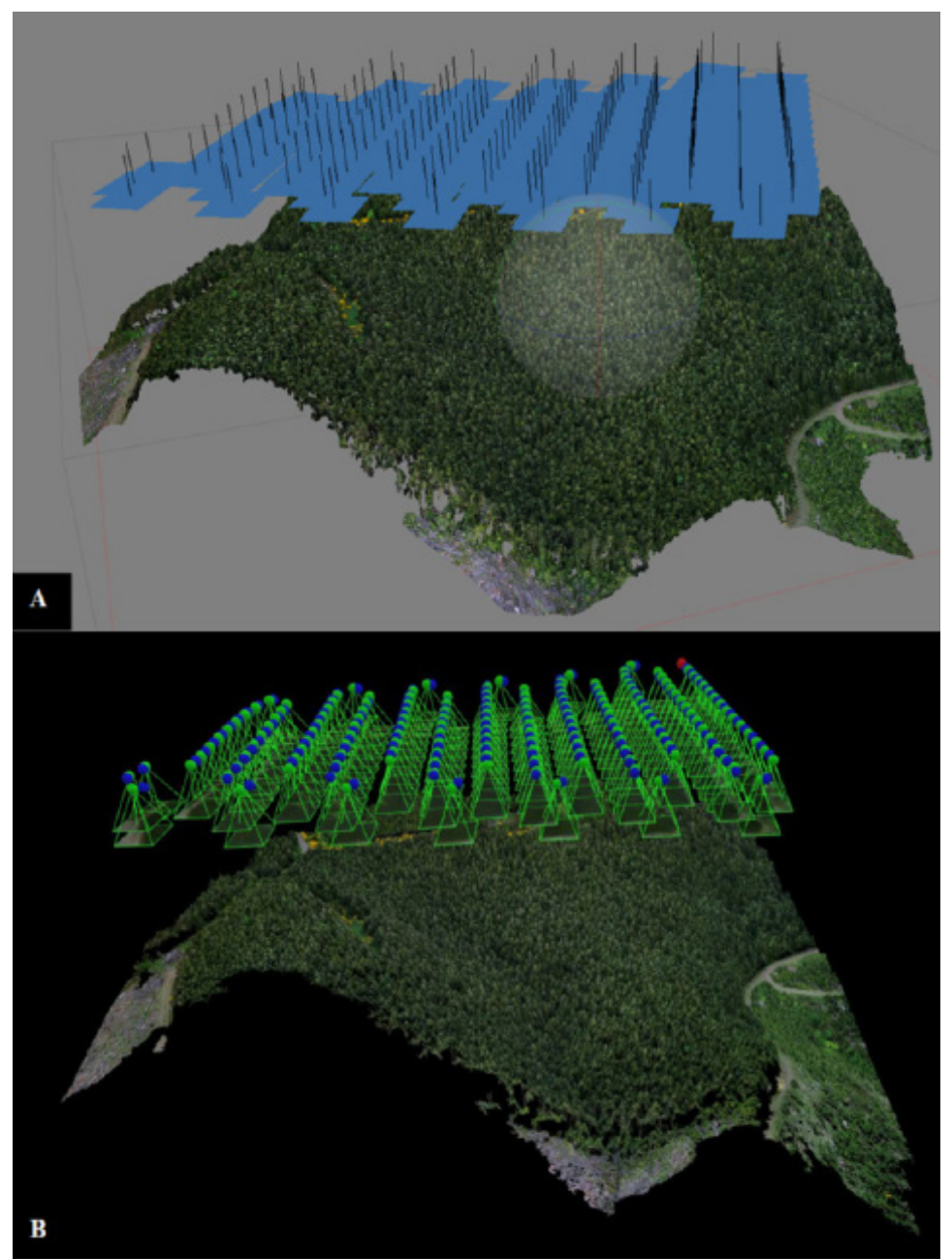

Figure 2 Point clouds produced by Agisoft (A) and Pix4D (B) eter was inactive) generic and reference preselection, Key point limit $(40,000)$, Tie point limit $(4,000)$, "checked" (indicating an active parameter) adaptive camera model fitting, and "aggressive" depth filtering. For Pix4D the fixed parameters were "checked" multiscale and minimum number of matches (3). Detailed explanations of Agisoft and Pix4D parameters and workflows are described in Agisoft (2018) and Pix4Dmapper (2018).

Eight (8) parameter combinations (four each from Agisoft and Pix4D) were used to generate point clouds. The point clouds generated in this study contained between 7 and 98 million points (Figure 2). Point clouds were cleaned of errors or outlier points. Error elimination was through investigation of the elevation histogram and visual identification of points significantly above canopy or below ground surface, referred to as pit or bird identification by some. Points identified as erroneous were deleted from the point cloud. The point clouds were normalized (i.e., ground elevation is subtracted from the elevation of each point) using ground profile estimation from a 10 $\mathrm{m}$ grid sampling using Quick Terrain Modeler (QTM) 8.07 software (QTM 2018) (Figure $3)$. The normalization process helped ensure accurate comparisons and was implemented in five stages: (i) lay out a grid of preset cell 


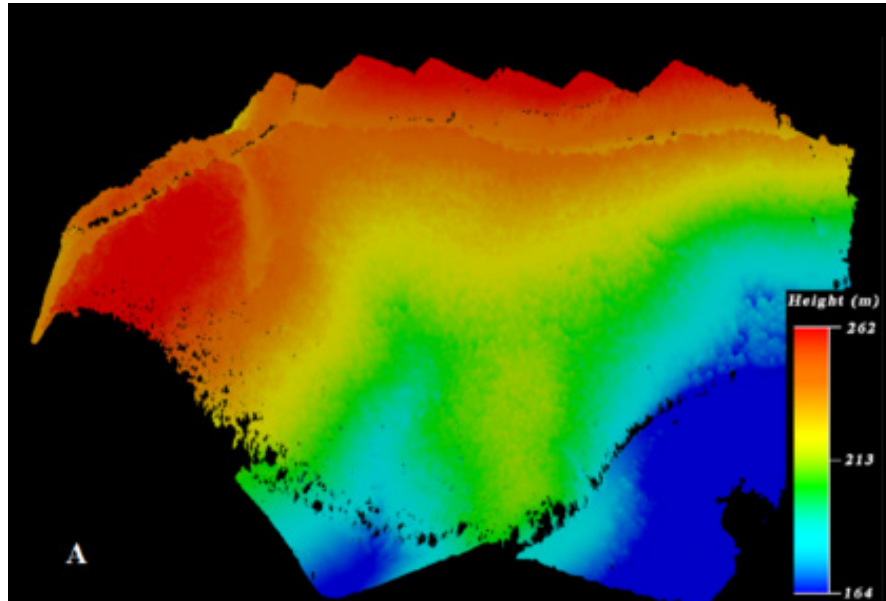

malized point clouds with the three tree segmentation algorithms: Variable Window Filter (VWF), Graph-Theoretical, and Watershed Segmentation. For comparison, trees that were manually segmented served as a comparative ground control to trees that were automatically delineated. Comparative ground control trees inside the sample plots were visually identified based on the normalized point clouds. Since the focus was on dominant and co-dominant trees, these trees were accurately identified by examining the point clouds from different angles.

\section{Variable Window Filter (VWF)}

The variable window filter (VWF) algorithm (Popescu \& Wynne 2004) uses a variable moving window to find the tree tops in a given CHM and is implemented in the ForestTools (R Package). The CHM was derived from normalized point clouds using QTM software with a 0.4

size over the point cloud, (ii) eliminate points to calculate a minimum elevation surface, (iii) perform outlier rejection and spike removal on the minimum elevation surface, (iv) filter the original points against the minimum elevation surface but keep any points close to the minimum elevation surface, (v) rebuild a new surface from the points resulting from stage (iv).

\section{Tree segmentation}

Individual trees were automatically delineated from the CHM generated from the nor- meter resolution. The moving window size varies depending on the tree height which assumes taller trees have wider crowns and vice versa. Thus, the linear relationship between height, which is a pixel value in a CHM, and window size needs to be specified in this algorithm. The linear model between height (x) and radius of the moving window (y) was initially specified using a default example from the ForestTools Package in R (i.e., $y=x \cdot 0.05+0.6$ ) (Plowright 2018). In addition, after testing for several height thresholds, two (2) meters was 
selected because excluding CHM cells lower than two meters seemed to produce better results in identifying dominant and co-dominant trees. The two meter height threshold was also intended to prevent the VWF algorithm from detecting bushes and shrubs.

\section{Graph-Theoretical (TrEx software)}

The TrEx algorithm identifies individual trees using an oriented weighted graph that is partitioned according to a set of weights defined by stand structure. The input was a grey-level image similar to a CHM generated in QTM software with 0.4 meter resolution. The difference is that Greyscale and Reverse Colors were selected in sequence for the default palettes option. In addition, the applied texture was selected before saving the file as a grey-level image. In TrEx software, after testing for different combinations, the values of the parameters that worked best with the stand structures and data were selected as follows. The settings for level count and level cutoff of 32 and 31, respectively, were selected as part of classification process for Pix4D point clouds. While for point clouds generated with Agisoft, level count and level cutoff were set to 62 and 61, respectively. The next step in TrEx was to create the hierarchies, with an adjacency value of four cells being selected.

TrEx also has adjustable cohesion criteria that determine whether multiple tree crown components are part of the same tree or not. Values chosen for weighted graph that balance the cohesion criteria were $50,30,0,20$, and 0 for level degree, node degree, surface, top distance, and centroid distance respectively. For partitioning, the keep best sufficient parent option was selected with threshold equal to 0.9 . In addition, common patches to closest tree was selected as the build trees option, and a filter by area value of 10 was used. A detailed explanation of TrEx algorithm parameters and workflow can be found in Strîmbu \& Strîmbu (2015).

\section{Watershed Segmentation}

Watershed Segmentation is one of three segmentation algorithms that can be used to delineate individual tree crown and estimate tree height (Goerndt et al. 2010, Edson \& Wing, 2011). The Watershed Segmentation algorithm is based on hydrological modelling tools implemented in ArcGIS 10.5.1. Tree crowns are identified as individual watersheds based on pixel height and resulting slopes determining the contributing area and the pixels therein that form a common watershed (crown) area.

The Watershed Segmentation delineates individual tree crowns and estimates tree height (Goerndt et al. 2010, Edson \& Wing 2011) by replacing the tree crowns, expressed as a canopy height model, with inversed basins. The input was a 0.4 meter resolution CHM generated in QTM software based on the normalized 3D point clouds. The CHM was processed into an inverse CHM using the raster calculator tool and used for subsequent processing (Figure 4).

\section{Algorithm assessment}

\section{Assessment plots}

The quality of the segmentation and the effect of the image processing algorithms on forest structure estimation were assessed by comparing computed trees with actual trees manually identified in nine 0.05 ha plots (Figure 5).

Trained analysts used point clouds and orthoimagery to delineate the location and height of all trees within each plot (Table 1). To account for trees that were close to the outside of a plot border, a two meter buffer was added to each plot. The plots were systematically distributed across the study area using a grid sampling method with equal distance between plots set at $80 \mathrm{~m}$. Systematic sampling was used to ensure the representativeness of all spatial and structural variation in the study area. 


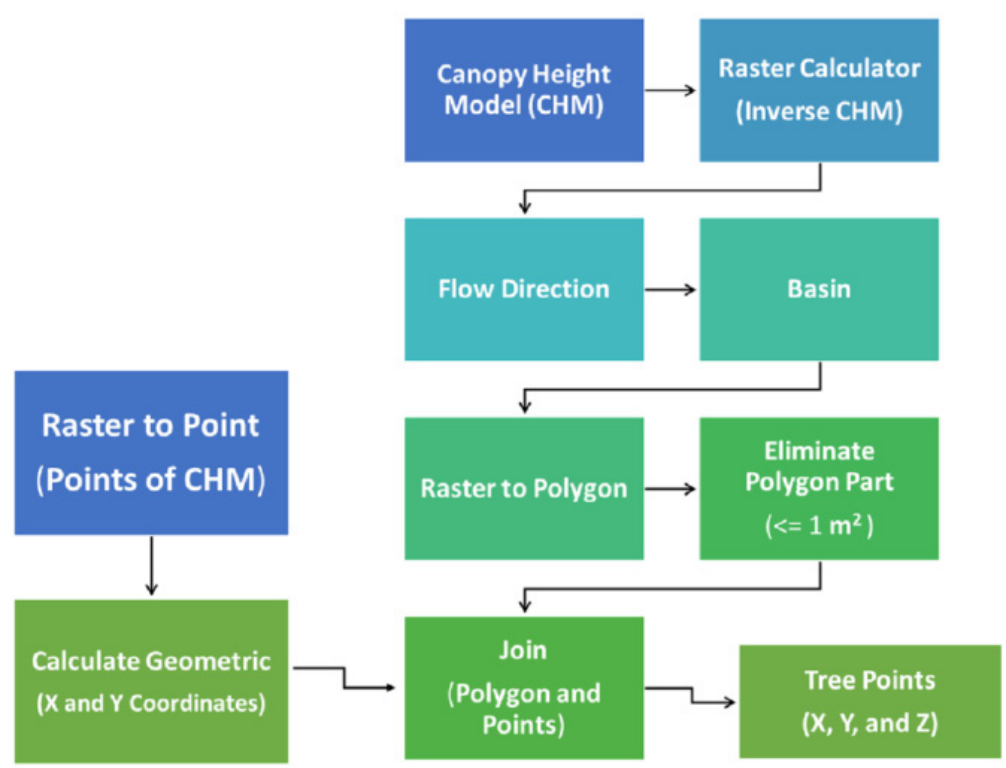

Figure 4 Watershed Segmentation work flow in ArcMap

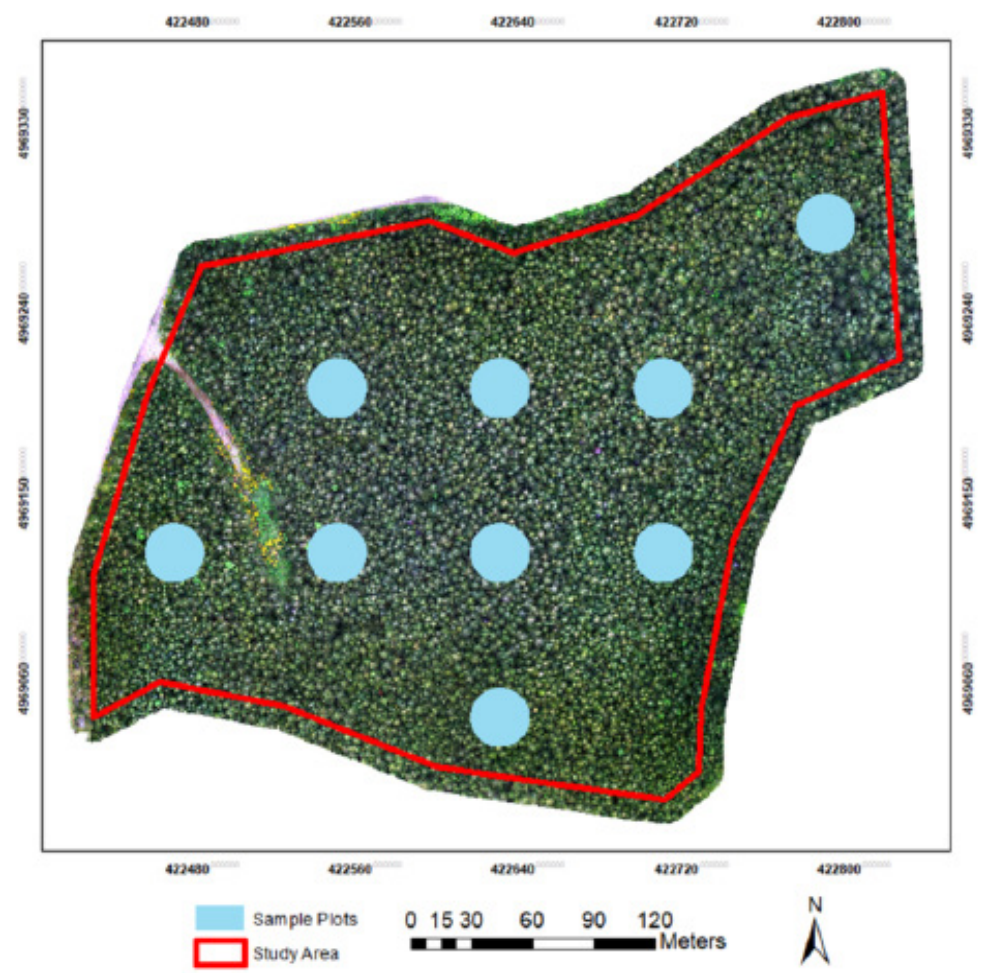

Figure 5 Sample plots distribution in the study area
Statistics for comparison of the algorithms

All comparisons between computed and manually segmented trees were done at plot level. The performance of each algorithm was assessed using eight statistics calculated as a percentage: (1) matched trees - M, (2) omission - O, (3) commission - C, (4) total error of number of trees - TEN, (5) accurate tree height - ATH, (6) underestimation of tree height - UTH, (7) overestimation of tree height - OTH, and (8) total error of tree height - TETH. Omission error is the actual trees not identified by the tree segmentation algorithms. Commission error, also known as false positive, is the additional trees misidentified by the tree segmentation algorithms. Furthermore, computed tree height was considered accurate if the estimation was between \pm 10 $\mathrm{cm}$ from the manual tree height. The eight statistics were calculated based on these following equations: 
Table 1 Number of trees and tree height within study plots

\begin{tabular}{lll}
\hline Plot & Number of trees & Mean tree height (meter) \\
\hline 1 & 70 & 6.04 \\
\hline 2 & 69 & 5.63 \\
\hline 3 & 61 & 7.91 \\
\hline 4 & 53 & 6.55 \\
\hline 5 & 71 & 6.18 \\
\hline 6 & 66 & 6.33 \\
\hline 7 & 62 & 6.58 \\
\hline 9 & 44 & 8.30 \\
\hline 9 & 59 & 7.79 \\
\hline
\end{tabular}

$O=($ Algorithm Omission $/$ MNT) $\cdot 100$

$C=($ Algorithm Commission $/$ MNT) $\cdot 100$

TEN $=(($ Algorithm Omission + Algorithm Commission)/MNT) $\cdot 100$

$M=1-(($ Algorithm Omission + Algorithm Commission) $/$ MNT) $\cdot 100$

UTH $=($ Algorithm Underestimation of Tree Height / MTH) $\cdot 100$

$\mathrm{OTH}=($ Algorithm Overestimation of Tree Height $)$ MTH) $\cdot 100$

TETH $=(($ Algorithm Underestimation + Overestimation of Tree Height)/MTH) 100

ATH $=(1-(($ Algorithm Underestimation + Overestimation of Tree Height)/MTH) ) 100

where: MNT - Manual Number of Trees, MTH Manual Tree Height.

Statistical analysis

The eight statistics for comparison of the algorithms were analyzed with a general linear model (GLM) ANOVA using SAS 9.4 (SAS 2018). In total, there were 216 observations; each tree segmentation algorithm has 72 observations (i.e., 9 plots $x 8$ point clouds). The analysis assessed tree segmentation algorithms and identified image processing algorithm parameters that had significant effects on forest structure estimation. The variables considered in ANOVA were the parameters defining each point cloud generation (Agisoft and Pix4D) and the three tree segmentation algorithms (i.e., VWF, TrEx, and Watershed). The general equation used for analysis was:

$Y=X_{1}+X_{2}+\varepsilon$

where: $Y$ - the estimated attributes, such as number of trees/plot or mean tree height/plot. $\mathrm{X}_{1}$ is the algorithm with two values for Pix4D and Agisoft, $\mathrm{X}_{2}$ is the tree segmentation algorithm with three values for VWF, TrEx, and Watershed Segmentation, the error term is denoted by $\varepsilon$. The parameters defining each algorithm were also assessed with the same general formula. Parameters defining Agisoft were image alignment with two values (high and medium) and dense point quality with two values (high and medium). Similar to Agisoft, Pix4D has two sets of parameters: image scale with two values (original and half) and point density with two values (optimal and low).

\section{Results}

The three tree segmentation algorithms that were applied to all point clouds generated 
with Agisoft and Pix4D resulted in 216 observations that were statistically analyzed using GLM. The results of the GLM analysis provided strong evidence that image processing algorithms can have significant impacts on forest structure estimation. However, parameters of the image processing algorithms that have significant effects on forest structure estimation varied.

\section{Assessment of Tree Identifying Algorithms}

Except for number of matched trees and total error of number of trees for Agisoft, all GLMs were significant which generally indicates significant differences among tree segmentation algorithm and image processing algorithm outputs (Table 2).

Assessment of algorithms identifying trees from agisoft point clouds

Variables indicating tree segmentation algorithms and image processing algorithm parameters in Agisoft were added as independent variables in GLM testing. The Agisoft parameters were image alignment (high and medium) and dense point quality (high and medium). Dense point quality parameter and tree segmentation algorithms had a significant effect on omission and commission (Table 3). Nevertheless, all Agisoft image processing algorithm parameters did not significantly affect the number of matched trees and total error of number of trees. In addition, image alignment parameter did not have a significant effect on all four tree identification statistics.

Matched and total error of number of trees were not significantly different between the tree segmentation algorithms and Agisoft parameters although some variation existed (Figure 6). The highest percent number of matched trees $(88.82 \%)$ was obtained by the Tree Extraction (TrEx) algorithm implemented from a from a point cloud with the "high" option for both image alignment and dense point quality parameters. The highest percent number of matched trees for Variable Window Filter (VWF) algorithm was 87.26\% when implemented from a CHM generated from a point cloud with the "high" option for image alignment parameter and "medium" option for dense point quality parameter. The watershed segmentation algorithm had the highest percent number of matched trees $(87.01 \%)$ when applied to a point cloud with the "medium" op-

Table 2 Number of trees and tree height within study plots

\begin{tabular}{llll}
\hline \multirow{2}{*}{ Statistic } & $p$ value & & \\
\cline { 2 - 4 } & Agisoft & Pix4D & Overall algorithms \\
\hline Matched & 0.43 & $* * *$ & $* * *$ \\
\hline Omission & $* * *$ & $* * *$ & $* * *$ \\
\hline Commission & $* * *$ & $* * *$ & $* * *$ \\
\hline
\end{tabular}

Note. The level of significance: $* * * p<0.0001$.

Table 3 General linear model of tree identification statistics for Agisoft point clouds

\begin{tabular}{lllll}
\hline \multirow{2}{*}{ Statistic } & $p$ value & & & \\
\cline { 2 - 5 } & Image alignment & Dense point quality & Tree segmentation algorithms & Plot \\
\hline Matched & 0.79 & 0.64 & 0.84 & 0.18 \\
\hline Omission & 0.97 & $* * *$ & $* * *$ & $* * *$ \\
\hline Commission & 0.69 & $* * *$ & $* *$ & $* * *$ \\
\hline
\end{tabular}

Note. The level of significance: $* * p<0.001, * * * p<0.0001$. 
tion for both image alignment and dense point quality parameter.

Omission and commission were significantly affected by dense point quality parameter and tree segmentation algorithms. Hence, variations existed in omission and commission for the combination of algorithms (Figure 6). In terms of omission, VWF performed the best with no omissions when it was implemented in a point cloud with the "high" option for both image alignment and dense point quality parameter, yet it had a $19.63 \%$ commission. TrEx had no commission error with the "high" option for image alignment and the "medium"

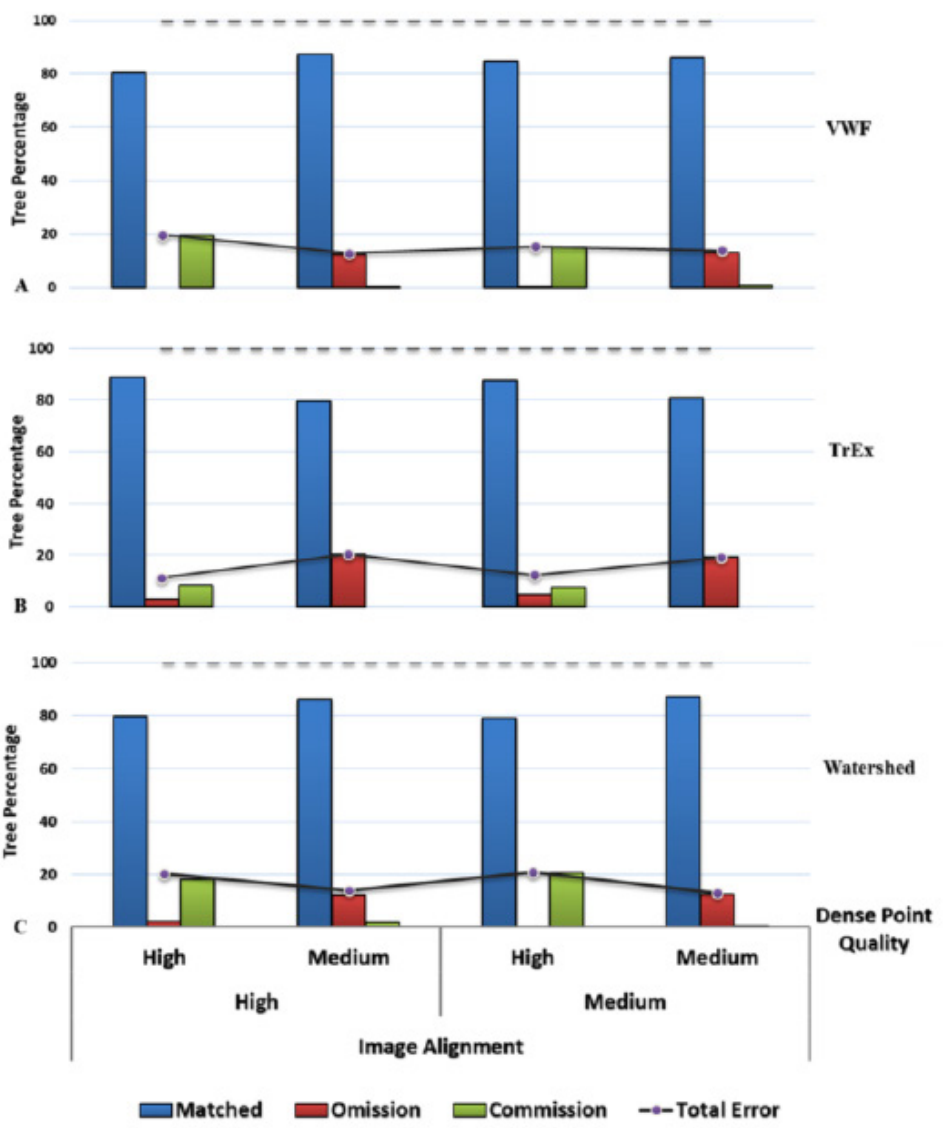

Figure 6 Assessment of tree segmentation algorithms in identifying tress with point clouds generated by Agisoft: Variable Window Filter (VWF), Tree Extraction (TrEx), and Watershed Segmentation option for dense point quality parameter but had around $20 \%$ omission. This demonstrates that an algorithm combination could have low omission with high commission and vice versa.

Assessment of algorithms identifying trees from Pix4D point clouds

Variables indicating tree segmentation algorithms and image processing algorithm parameters in Pix4D were added as independent variables in a GLM. For Pix4D, tree segmentation algorithms had a significant effect on all tree identification statistics (Table 4). The image scale parameter also had a significant impact on all tree identification statistics with the exception of omission. In contrast, point density parameter appeared to have no significant effect.

Variation existed in the percent of matched trees for each combination of tree segmentation and Pix4D image processing algorithm parameters (Figure 7). TrEx outperformed VWF and Watershed Segmentation in the four Pix4D point clouds with percent matched trees between $83.39 \%$ and $89.15 \%$. TrEx had the highest percent matched trees when it was implemented in the CHM derived from a Pix4D point cloud with "original" and "optimal" option for image scale and point density parameters, respectively. The high- 
Table 4 General linear model of tree identification statistics for Pix4D point clouds

\begin{tabular}{lllll}
\hline \multirow{2}{*}{ Statistic } & $p$ value & & & \\
\cline { 2 - 5 } & Image scale & Point density & Tree segmentation algorithms & Plot \\
\hline Matched & $* *$ & 0.50 & $* * *$ & $* * *$ \\
\hline Omission & 0.89 & 0.07 & $* * *$ & $* *$ \\
\hline Commission & $* *$ & 0.27 & $* * *$ & $* * *$ \\
\hline
\end{tabular}

Note. The level of significance: ${ }^{* *} p<0.001,{ }^{* * *} p<0.0001$

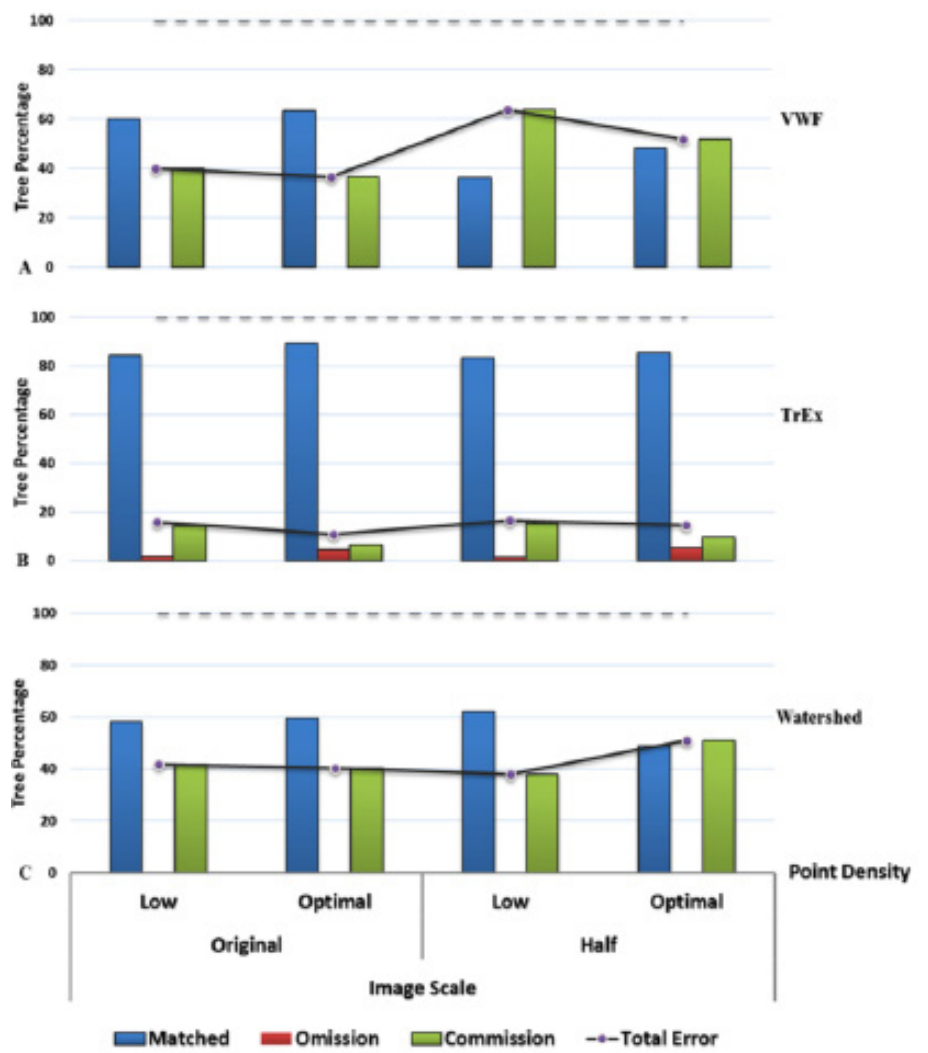

Figure 7 Assessment of tree segmentation algorithms in identifying tress with point clouds generated by Pix4D: Variable Window Filter (VWF), Tree Extraction (TrEx), and Watershed Segmentation est percent matched trees for VWF $(63.43 \%)$ was also determined in the same parameter configuration. In addition, the highest percent matched trees for watershed segmentation $(62 \%)$ was obtained with "half" image scale and "low" point density parameters.
Assessment of algorithms identifying trees from overall point clouds

Tree segmentation algorithms (i.e., VWF, TrEx, and Watershed Segmentation) and point cloud generation algorithms (i.e., Agisoft and Pix4D) were added as independent variables in a GLM with tree identification statistics as dependent variables. The results showed that tree segmentation algorithms and point cloud generation algorithms had significant effects on the four tree identification statistics (Table 5).

The three tree segmentation algorithms had similar high percentages of matched trees in Agisoft point clouds and strong consistency with percentages between $83 \%$ and $84.61 \%$ (Figure 8 ). In contrast, tree segmentation algorithms did not perform well in identifying trees in the Pix4D point clouds with the exception of TrEx. On average, TrEx achieved $85.51 \%$ matched trees when implemented with Pix4D point clouds, while VWF and Watershed Segmentation had percent matched trees lower than $60 \%$. In 
Table 5 General linear model of tree identification statistics for overall point clouds

\begin{tabular}{llll}
\hline \multirow{2}{*}{ Statistic } & \multicolumn{2}{l}{$p$ value } & \\
\cline { 2 - 4 } & Point clouds generation algorithms & Tree segmentation algorithms & Plot \\
\hline Matched & $* * *$ & $* * *$ & $* * *$ \\
\hline Omission & $* * *$ & $* * *$ & $* *$ \\
\hline Commission & $* * *$ & $* * *$ & $* * *$ \\
\hline
\end{tabular}

Note. The level of significance: $* * p<0.001, * * * p<0.0001$.

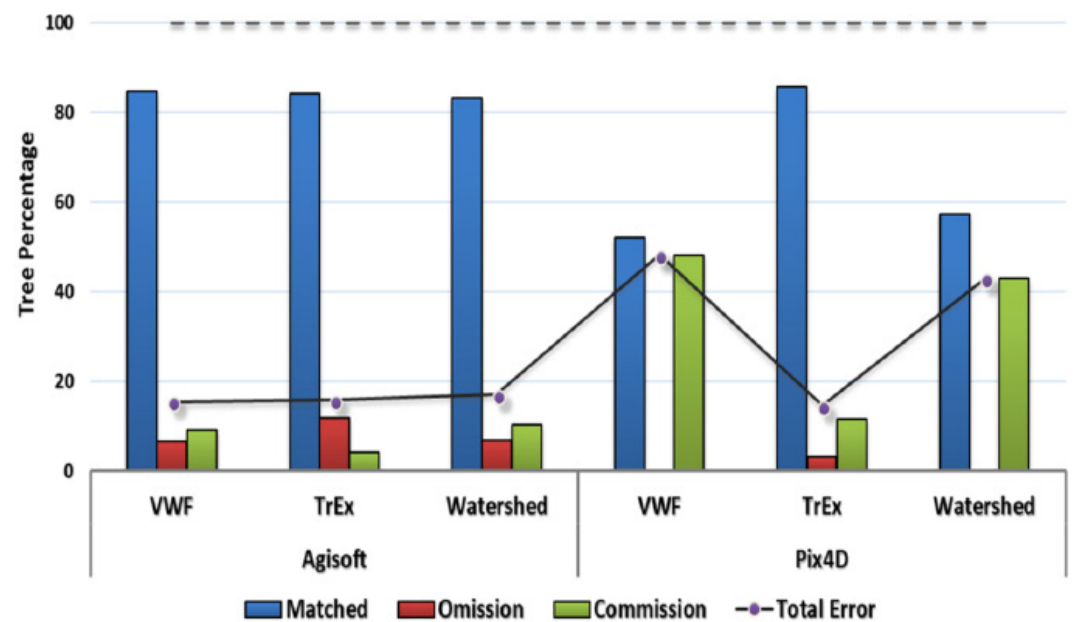

Figure 8 Assessment of tree segmentation algorithms in identifying tress

terms of omission and commission, tree segmentation algorithms applied with Agisoft point clouds had relatively balanced omission and commission results between $4 \%$ and $12 \%$. For Pix4D point clouds, TrEx performed relatively well but the other two algorithms had considerably high commission errors ranging from $42 \%$ to $48 \%$. The good performance of TrEx with both type of point clouds (i.e., Agisoft and Pix4D) indicates a robust algorithm.

Overall, five algorithms and parameter combinations had the highest number of matched trees (Table 6). The column labelled "Algorithm Parameter" in Table 6 contains acronyms of the algorithm parameters used for Agisoft and Pix4D. Pix4D produced the highest percent matched trees, Agisoft claimed the next four spots, but all percentages were within approximately $2 \%$ of one another.

\section{Assessment of algorithms estimating tree height}

To assess tree height estimation, four statistics were calculated as a percentage: (1) accurate tree height, (2) underestimation of tree height, (3) over estimation of tree height, and (4) total error of tree height. Computed tree heights were considered accurate if the estimation was between $\pm 10 \mathrm{~cm}$ from the manual tree height measurement. Overall, GLM results were significant indicating strong evidence that means of the four tree height estimation statistics were significantly different between tree segmentation and image processing algorithms (Table 7).

Assessment of algorithms estimating tree height from agisoft point clouds

Strong evidence existed that the dense point quality parameter significantly affected tree height estimation (Table 8). Contrarily, tree segmentation algorithms seem to have no significant effect in estimating tree height from Agisoft point clouds. In addition, the image 
alignment parameter had a significant effect in overestimation of tree height (P-value: < 0.001 , yet did not significantly affect other tree height estimation statistics (Table 8). Interestingly, the plot variable seems to have a significant impact on tree height estimation. Although it is beyond the scope of this study, the significant effect of the sample plots may be due to spatial and structural variation among sample plot areas.

The tree segmentation algorithms appeared to have similar patterns in estimating tree height from Agisoft point clouds (Figure 9).
Tree segmentation algorithms implemented in Agisoft point clouds generated with the "high" option for dense point quality had higher percent accurate tree heights with a range from $96.50 \%$ to $99.33 \%$. The highest percentage was achieved by VWF with an Agisoft point cloud with the "high" option for both image alignment and dense point quality parameters.

All tree segmentation algorithms had a very small percent underestimation of tree heights when applied with Agisoft point clouds with a "high" dense point quality parameter; the percent underestimation varied from $0.42 \%$

Table 6 Five algorithms combinations with the highest percent matched trees

\begin{tabular}{llll}
\hline $\begin{array}{l}\text { Algorithm* } \\
\text { parameter }\end{array}$ & $\begin{array}{l}\text { Matched } \\
\text { trees (\%) }\end{array}$ & Point cloud generation algorithm & Tree segmentation algorithm \\
\hline OO & 89.16 & Pix4D & TrEx \\
\hline HH & 88.82 & Agisoft & TrEx \\
\hline MH & 87.63 & Agisoft & TrEx \\
\hline HM & 87.26 & Agisoft & VWF \\
\hline MM & 87.01 & Agisoft & Watershed \\
\hline
\end{tabular}

Note. * For Agisoft, the letters in column 1 idicate image alignment parameter (i.e., high $\mathrm{H}$ or medium $\mathrm{M}$ ) and dense point quality parameter (i.e., high $\mathrm{H}$ or medium $\mathrm{H}$ ) respectively. For Pix $4 \mathrm{D}$, the letters indicate image scale parameter (i.e., original $\mathrm{O}$ or half $\mathrm{H}$ ) and point density parameter (optimal $\mathrm{O}$ or low $\mathrm{L}$ ) respectively.

Table 7 General linear model of tree height estimation statistics

\begin{tabular}{llll}
\hline \multirow{2}{*}{ Statistic } & $p$ value & & \\
\cline { 2 - 4 } & Agisoft & Pix4D & Overall algorithms \\
\hline Accurate tree height & $* * *$ & $* * *$ & $* * *$ \\
\hline Underestimation of tree height & $* * *$ & $* * *$ & $* * *$ \\
\hline Overestimation of tree height & $* * *$ & $* * *$ & $* * *$ \\
\hline
\end{tabular}

Note. The level of significance: $* * * p<0.0001$.

Table 8 General linear model of tree height estimation statistics for Agisoft point clouds

\begin{tabular}{lllll}
\hline \multirow{2}{*}{ Statistic } & \multicolumn{2}{l}{$p$ value } & & \\
\cline { 2 - 5 } & Image alignment & Dense point quality & $\begin{array}{l}\text { Tree segmentation } \\
\text { algorithms }\end{array}$ & Plot \\
\hline Accurate tree height & 0.34 & $* * *$ & 0.97 & $* * *$ \\
\hline Underestimation of tree height & 0.68 & $* * *$ & 0.85 & $* * *$ \\
\hline Overestimation of tree height & $* *$ & $* * *$ & 0.75 & $*$ \\
\hline
\end{tabular}

Note. The level of significance: $* p<0.05 * *, p<0.001, * * * p<0.0001$. 
to $1.54 \%$. With the same type of point clouds, the tree segmentation algorithms had relatively small percent overestimations of tree height with a range from $0.10 \%$ to $2.19 \%$. As a result, the combination of these tree segmentation and image processing algorithm parameters had relatively small percent total error and high percent accurate tree heights. Moreover, despite demonstrating no height overestimation, the tree segmentation algorithms implemented in Agisoft point clouds generated with the "medium" dense point quality parameter tended to have a relatively high percent height underestimation between $11.22 \%$ and $13.23 \%$.
Assessment of algorithms estimating tree height from Pix4D point clouds

The image scale parameter had a significant effect on the four tree height estimation statistics (Table 9). Furthermore, the point density parameter significantly affected all four tree height estimation statistics except for the underestimation of tree height ( $p$ value: 0.09). As with the point density parameter, the tree segmentation algorithms also had a significant effect on height estimation except for underestimation. Sample plots also had a significant impact on tree height estimation.

The highest percent accurate tree heights $(94.62 \%)$ was achieved by the VWF algorithm from a Pix4D point cloud with the "half" image scale and "low" point density parameters (Figure 10). With the same type of point cloud, the Watershed Segmentation and TrEx algorithms obtained almost the same percent accurate tree height $(\sim 94.41 \%)$. All tree segmentation algorithms resulted in a relatively low percent height underestimation (< 2.1\%).However, overestimation varied considerably among the segmentation and processing algorithm parameters (3\% to $15 \%$ ).

Figure 9 Tree height estimation by tree segmentation algorithms with point clouds generated by Agisoft: Variable Window Filter (VWF), Tree Extraction (TrEx), and Watershed Segmentation 
Assessment of algorithms estimating tree height from overall point clouds

There is strong evidence that point clouds generation algorithms and sample plots had a significant impact on tree height estimation
(Table 10). Contrarily, tree segmentation algorithm choice did not significantly affect tree height estimation.

In general, all the tree segmentation algorithms had relatively similar high percent accurate tree height in Agisoft point clouds

Table 9 General linear model of tree height estimation statistics for Pix4D point clouds

\begin{tabular}{lllll}
\hline \multirow{2}{*}{ Statistics } & \multicolumn{2}{l}{$p$ value } & & \\
\cline { 2 - 6 } & Image scale & Point density & Tree segmentation algorithms & Plot \\
\hline Accurate tree height & $* * *$ & $* * *$ & $*$ & $* * *$ \\
\hline Underestimation of tree height & $*$ & 0.09 & 0.39 & $* * *$ \\
\hline Overestimation of tree height & $* * *$ & $* * *$ & $* * *$ & $* * *$ \\
\hline
\end{tabular}

Note. The level of significance: $* p<0.05, * * * p<0.0001$.
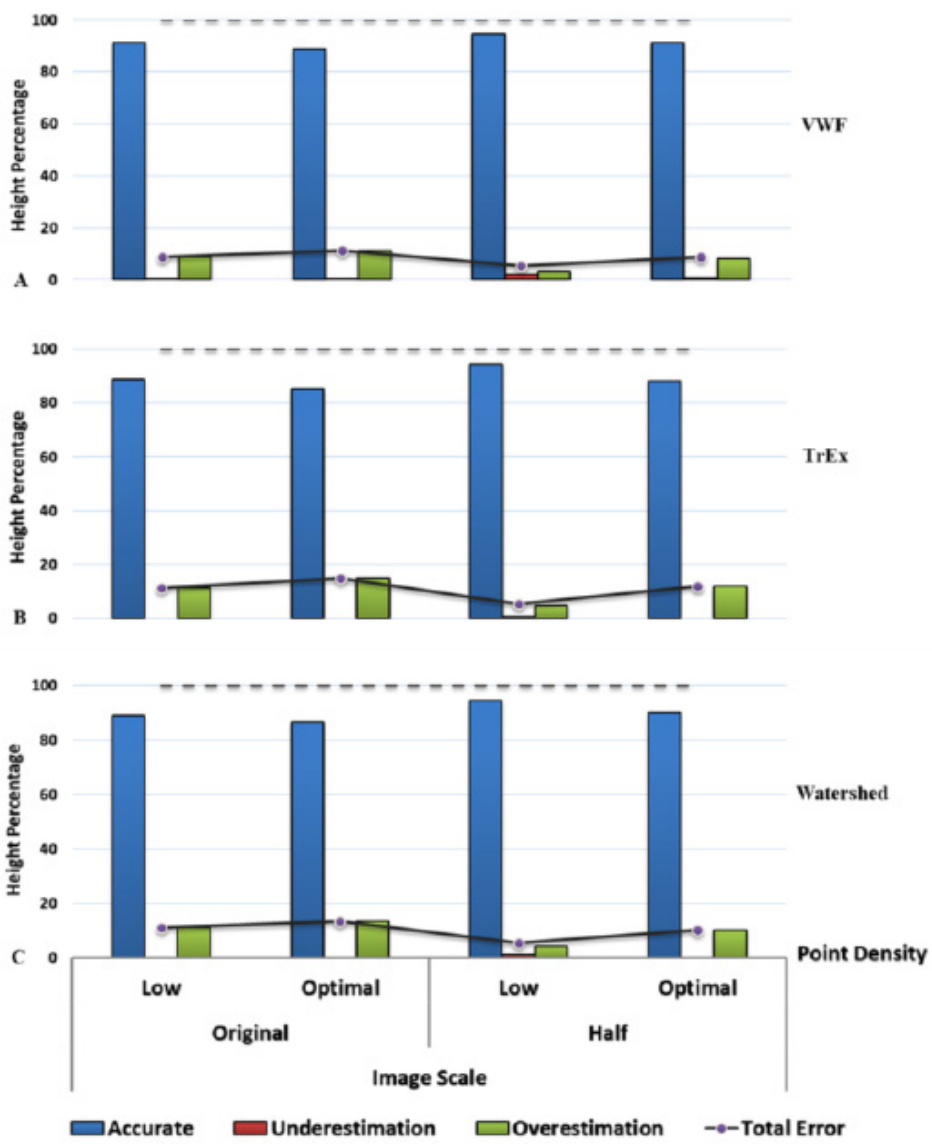

Figure 10 Tree height estimation by tree segmentation algorithms with point clouds generated by Pix4D: Variable Window Filter (VWF), Tree Extraction (TrEx), and Watershed Segmentation
( $93 \%)$. The highest percent accurate tree height for Pix4D (91.46\%) was achieved by the VWF algorithm. Watershed segmentation and TrEx algorithms had $89.98 \%$ and $89.02 \%$ accurate tree height respectively for Pix4D. Regarding underestimation and overestimation of the tree height, the tree segmentations algorithms performed differently with Agisoft and Pix4D point clouds. The tree segmentation algorithms tended to have a higher percent underestimation of tree height with Agisoft point clouds of around $7 \%$, yet they had a low percent overestimation of less than $1 \%$. For comparison, tree height segmentation algorithms had a higher percent overestimation when implemented in 
Pix4D point clouds with percent overestimation between $8 \%$ and $11 \%$. However, they had a relatively low percent underestimation of tree height of less than $1 \%$.

The five algorithm combinations that had the highest percent accurate tree height were all created with Agisoft generated point clouds (Table 11). All three tree height estimating algorithms were represented in the top five.

\section{Discussion}

\section{Tree identification}

In general, except for the number of matched trees and total error of number of trees from Agisoft point clouds, there is strong evidence that image processing algorithms and tree segmentation algorithms have a significant effect on forest structure estimation (Table 2).Using a LiDAR dataset, Edson \& Wing (2011) and Kaartinen et al. (2012) also found that different tree segmentation algorithms can have different results in estimating number of trees.

Particularly for Agisoft point clouds, there were variations of percent matched trees and total error of the number of trees (Figure 6). However, tree segmentation and image processing algorithm parameters did not have a significant effect on the percent of matched trees or total error of tree numbers (Table 3). For the tree segmentation algorithms, the percent matched trees achieved with Agisoft point clouds was consistent and ranged from 83\% to $85 \%$. Edson \& Wing (2011) examined a LiDAR dataset and obtained noticeably different results between Watershed Segmentation and TreeVaW which is similar to the VWF algorithm. In their study, Watershed Segmentation and TreeVaW achieved 93\% and 59\% number of matched trees, respectively.

In terms of omission and commission, however, dense point quality and tree segmentation algorithms do appear as significant effects for Agisoft (Table 3). This is likely because a

Table 10 General linear model of tree height estimation statistics for overall point clouds

\begin{tabular}{|c|c|c|c|}
\hline \multirow[b]{2}{*}{ Statistic } & \multicolumn{3}{|l|}{$p$ value } \\
\hline & $\begin{array}{l}\text { Point clouds generation } \\
\text { algorithms }\end{array}$ & $\begin{array}{l}\text { Tree segmentation } \\
\text { algorithms }\end{array}$ & Plot \\
\hline Accurate tree height & $* *$ & 0.52 & $* * *$ \\
\hline Underestimation of tree height & $* * *$ & 0.87 & $*$ \\
\hline Overestimation of tree height & $* * *$ & 0.11 & $* * *$ \\
\hline
\end{tabular}

Table 11 General linear model of tree height estimation statistics for overall point clouds

\begin{tabular}{llll}
\hline $\begin{array}{l}\text { Algorithm* } \\
\text { Parameters }\end{array}$ & $\begin{array}{l}\text { Accurate Tree Height } \\
(\%)\end{array}$ & $\begin{array}{l}\text { Point Cloud Generation } \\
\text { Algorithm }\end{array}$ & $\begin{array}{l}\text { Tree Segmentation } \\
\text { Algorithm }\end{array}$ \\
\hline $\mathrm{HH}$ & 99.33 & Agisoft & VWF \\
\hline $\mathrm{HH}$ & 98.99 & Agisoft & Watershed \\
\hline $\mathrm{HH}$ & 98.76 & Agisoft & TrEx \\
\hline $\mathrm{MH}$ & 96.85 & Agisoft & Watershed \\
\hline $\mathrm{MH}$ & 96.57 & Agisoft & VWF \\
\hline
\end{tabular}

Note. * For Agisoft, the letters idicate image alignment parameter (i.e., high (H) or medium (M)) and dense point quality parameter (i.e., high $\mathrm{H}$ or medium $\mathrm{H}$ ) respectively. For Pix4D, the letters indicate image scale parameter (i.e., original $\mathrm{O}$ or half $\mathrm{H}$ ) and point density parameter (optimal $\mathrm{O}$ or low $\mathrm{L}$ ) respectively. 
combination of tree segmentation and data processing algorithm that produces high omission errors will also tend to have low commission errors and vice versa (Figure 6).

For Pix4D, image scale parameter and tree segmentation algorithms significantly affected the number of matched trees (Table 4). There was a noticeable difference in the percent number of matched trees between the three tree segmentation algorithms (Figure 7). Among the tree segmentation algorithms, only TrEx performed relatively well with Pix4D point clouds. While both VWF and Watershed Segmentation algorithms obtained percent matched trees below $64 \%$, most of the errors were commission. This was an interesting finding, since VWF, Watershed Segmentation, and TrEx all had relatively equal high percent matched trees in Agisoft point clouds. This could be a consequence of the high level of detail captured in Pix4D point clouds. When a point cloud captures enhanced details of an individual tree, the digital tree structure may result in branches that are identifiable and distinguished from the main stem potentially leading to an erroneously identified tree (i.e. commission error). Enhanced point cloud detail could potentially be advantageous if the tree segmentation algorithm were robust and adjustable with the stand characteristics as demonstrated by the TrEx algorithm. Otherwise, a high commission error by a tree segmentation algorithm may result.

When all the observations from Agisoft and Pix4D were combined and analyzed using a GLM, we found that image processing algorithms used by Agisoft and Pix4D have significant effects on tree identification (Table 5). The different image processing algorithms implemented in Agisoft and Pix4D led to a significantly different number of estimated trees. This demonstrated that Agisoft point clouds captured sufficient detail to allow tree segmentation algorithms to identify trees. In addition, based on visual observation, Agisoft seems to simplify the shape of the trees to look like cones which likely makes it easier for a tree segmentation algorithm to identify the trees. While Pix4D point clouds seem to have greater detail in representing trees, this enhanced detail potentially leads to greater error depending on the segmentation algorithm.

Besides image processing algorithms, tree segmentation algorithm choice had an important role in tree identification (Table 5). In general, we found that Agisoft performed admirably as all tree segmentation algorithms tested had a high percent number of matched trees. For tree segmentation algorithms, TrEx appeared to perform most strongly as it had consistent and high percent number of matched trees with both Agisoft and Pix4D point clouds.

\section{Tree height estimation}

In general, GLM results found significant differences in tree height estimation between different tree segmentation and data processing algorithm parameters. For Agisoft parameters, only the dense point quality parameter appeared to have a significant effect on the percentage of matched trees (Table 7). This emphasizes that quality or the density of the point cloud significantly affects tree height estimation. For Agisoft point clouds, the highest percent accurate tree height was achieved by the VWFwhen implemented on a point cloud generated with the "high" option for image alignment and dense point quality. Wallace et al. (2016) also used this combination and found that forest structure estimation from photogrammetric point clouds was comparable to LiDAR point clouds.

For Pix4D, the VWF achieved the highest accuracy when implemented with a "half" image scale and "low" point density (Table 8). This demonstrates that increasing the quality of image scale parameter from "half" to "original" and point density parameter from "low" to "optimal" does not necessarily result in higher tree height accuracies. 
Nevertheless, the tree segmentations algorithms seemed to have relatively consistent results in estimating tree height from Agisoft and Pix4D point clouds. The percent accurate tree height ranged from $86 \%$ to $99 \%$ for Agisoft and $85 \%$ to $95 \%$ for Pix $4 \mathrm{D}$.

\section{Conclusions}

We assessed the sensitivity of forest structure estimation to UAS-based image processing algorithms. For comparison, different image processing algorithm parameters from Agisoft and Pix4D were used to generate point clouds. In addition, the performance of tree segmentation algorithms (i.e., Variable Window Filter (VWF), Tree Extraction (TrEx), and Watershed Segmentation) implemented in different image processing algorithms was also evaluated in this study.

In general, the type of image processing algorithms and tree segmentation algorithms appeared to have a significant effect on tree identification. For Agisoft, however, the image processing algorithm parameters and tree segmentation algorithms used in this study appeared to have no significant effect on the percent number of matched trees. All three tree segmentation algorithms achieved a relatively high percentage of matched trees from Agisoft point clouds. This likely resulted from high quality and high density of the Agisoft point clouds produced in this study. In addition, based on visual observation, the shape of the trees represented in the Agisoft point clouds seem to be simplified into a shape that approximates a cone, which likely makes it easier for a tree segmentation algorithm to identify the trees. In contrast, Pix4D represents trees in a less regular and more diverse digital shape. However, in terms of omission and commission for Agisoft, the dense point quality parameter and tree segmentation algorithms had significant effects that resulted in some variation for omission and commission errors.
The Pix4D image scale parameter and tree segmentation algorithms significantly affected the number of matched trees. Interestingly, from the three segmentation algorithms used in this study, only TrEx had a relatively high percent number of matched trees from Pix4D point clouds. This might happen as a result of the highly detailed representation of trees that were captured by Pix4D. A detailed representation of the trees would be an advantage if the tree segmentation algorithm was robust and adjustable with the stand structure and dataset. Otherwise, the tree segmentation algorithm could misidentify an individual tree as multiple trees (commission error).

For tree height estimation, in general, there is strong evidence that image processing algorithms have significant effects. However, tree segmentation algorithms do not significantly affect tree height estimation. For Agisoft, the dense point quality parameter appeared to have a significant effect. The higher quality and higher density of the Agisoft point clouds resulted in a higher percent accurate tree height. For Pix4D, the image scale and point density parameters significantly affected tree height estimation. However, based on the parameters that were used in this study, using higher quality for image scale and point density does not necessarily increase percent accurate tree height since the highest percentage was achieved by variable window filter (VWF) algorithm that was implemented in a CHM derived from a Pix4D point cloud generated with "half" image scale and "low" point density.

It is important to choose an adequate data processing algorithm when estimating forest structure from high resolution remotely sensed data such as that typically collected by a UAS. Using a robust and adjustable tree segmentation algorithm is also recommended to achieve preferred results in forest structure estimation. This study also recommends some combinations of tree segmentation and data processing algorithms that relatively performed well in identifying trees and estimating tree height 
(Table 5 and Table 10). However, this study is limited to coniferous trees which are relatively easier to identify when compared to deciduous trees. To fully understand the effect of image processing algorithms and tree segmentation algorithms on forest structure estimation, it is recommended that future studies examine more image processing algorithm parameters and tree segmentation algorithms in a broad assortment of forests, including coniferous, deciduous, and mixed forests.

\section{References}

Abdollahnejad A., Panagiotidis D., SurovýP., 2018. Estimation and extrapolation of tree parameters using spectral correlation between UAV and Pléiades data. Forests 9(2): 85 . DOI: $10.3390 / \mathrm{f} 9020085$

Agisoft, 2018. Agisoft PhotoScan User Manual - Professional Edition, Version 1.3. Web: http://www.agisoft.com/pdf/photoscan-pro_1_3_en.pdf Accessed: 03.06.18

Carr J.C., Slyder J.B., 2018. Individual tree segmentation from a leaf-off photogrammetric point cloud. International Journal of Remote Sensing 39: 1-16. DOI: 10.1080/01431161.2018.1434330

Cook K.L., 2017. An evaluation of the effectiveness of low-cost UAVs and structure from motion for geomorphic change detection. Geomorphology 278: 195-208. DOI: 10.1016/j.geomorph.2016.11.009

Dandois J., Olano M., Ellis E., 2015. Optimal altitude, overlap, and weather conditions for computer vision UAV estimates of forest structure. Remote Sensing 7(10): 13895-13920. DOI: 10.3390/rs71013895

Edson C., Wing M.G., 2011. Airborne light detection and ranging (LiDAR) for individual tree stem location, height, and biomass measurements. Remote Sensing 3(12): 2494-2528. DOI: 10.3390/rs3112494

Fang R., Strimbu B.M., 2017. Stem measurements and taper modeling using hotogrammetric point clouds. Remote Sensing 9(7):716. DOI: 10.3390/rs9070716

Fernández-Guisuraga J., Sanz-Ablanedo E., Suárez-Seoane S., Calvo L., 2018. Using unmanned aerial vehicles in postfire vegetation survey campaigns through large and heterogeneous areas: Opportunities and challenges. Sensors 18(2): 586. DOI: 10.3390/s18020586

Goerndt M.E., Monleon V.J., Temesgen H., 2010. Relating forest attributes with area - and tree-based light detection and ranging metrics for Western Oregon. Western Journal of Applied Forestry 25(3): 105-111. DOI: 10.1093/wjaf/25.3.105

ICAO, 2011. Unmanned aircraft systems: (UAS). Web: https://www.icao.int/Meetings/UAS/Documents/Circu- lar\%20328 en.pdf Accessed: 13.02.17

Kaartinen H., Hyyppä J., Yu X., Vastaranta M., Hyyppä H., Kukko A., Holopainen M., HeipkeC., Hirschmugl M., Morsdorf F., Næsset E., Pitkänen J., Popescu S., Solberg S., Wolf B.M., Wu J., 2012. An International comparison of individual tree detection and extraction using airborne laser scanning. Remote Sensing 4(4): 950-974. DOI: 10.3390/rs4040950

Kachamba D., Ørka H., Gobakken T., Eid T., Mwase W., 2016. Biomass estimation using 3D data from Unmanned Aerial Vehicle imagery in a tropical woodland. Remote Sensing 8(11): 968. DOI: 10.3390/rs8110968

Méndez-Barroso L.A., Zárate-Valdez J.L., Robles-Morúa A., 2018. Estimation of hydromorphological attributes of a small forested catchment by applying the Structure from Motion (SfM) approach. International Journal of Applied Earth Observation and Geoinformation 69: 186-197. DOI: 10.1016/j.jag.2018.02.015

Messinger M., Asner G., Silman M., 2016. Rapid assessments of Amazon forest structure and biomass using small Unmanned Aerial Systems. Remote Sensing 8(8): 615. DOI: $10.3390 / \mathrm{rs} 8080615$

Mlambo R., Woodhouse I., Gerard F., Anderson K., 2017. Structure from Motion (SfM) Photogrammetry with drone data: A low cost method for monitoring greenhouse gas emissions from forests in developing countries. Forests 8(3): 68. DOI: 10.3390/f8030068

Ota T., Ogawa M., Mizoue N., Fukumoto K., Yoshida S., 2017. Forest structure estimation from a UAV-Based photogrammetric point cloud in managed temperate coniferous forests. Forests 8(9): 343. DOI: 10.3390/ f8090343

Ota T., Ogawa M., Shimizu K., Kajisa T., Mizoue N., Yoshida S., Takao G., Hirata Y., Furuya N., Sano T., Sokh H., Ma V., Ito E., Toriyama J., Monda Y., Saito H., Kiyono Y., Chann S., Ket N., 2015. Aboveground biomass estimation using structure from motion aproach with aerial photographs in a seasonal tropical forest. Forests 6(11): 3882-3898. DOI: 10.3390/f6113882

Panagiotidis D., Abdollahnejad A., Surový P., Chiteculo V., 2017. Determining tree height and crown diameter from high-resolution UAV imagery. International Journal of Remote Sensing 38(8-10): 2392-2410. DOI: 10.1080/01431161.2016.1264028

Paneque-Gálvez J., McCall M., Napoletano B., Wich S., Koh L., 2014. Small drones for community-based forest monitoring: An assessment of their feasibility and potential in tropical areas. Forests 5(6): 1481-1507. DOI: 10.3390/f5061481

Perroy R.L., Sullivan T., Stephenson N., 2017. Assessing the impacts of canopy openness and flight parameters on detecting a sub-canopy tropical invasive plant using a small unmanned aerial system. ISPRS Journal of Photogrammetry and Remote Sensing 125: 174-183. DOI: 10.1016/j.isprsjprs.2017.01.018

Pix4Dmapper, 2018. Pix4Dmapper 4.1 User Manual. Web: http://support.pix4d.com/hc/en-us/articles/204272989-Offline-Getting-Started-and-Manu- 
al-pdf- Accesses: 04.06.18

Plowright A., 2018. Canopy analysis in $\mathrm{R}$ using Forest Tools. Web: https://cran.r-project.org/web/packages/ ForestTools/vignettes/treetopAnalysis.html Accessed: 24.07.18

Popescu S.C., Wynne R.H., 2004. Seeing the trees in the forest: Using Lidar and multispectral data fusion with local filtering and variable window size for estimating tree height. Photogrammetric Engineering and Remote Sensing 70: 589-604. DOI: 10.14358/PERS.70.5.589

QTM, 2018. Tutorials - Applied Imagery. Web: http://appliedimagery.com/tutorials/. Accessed: 23.07.18

Roşca S., Suomalainen J., Bartholomeus H., Herold M., 2018. Comparing terrestrial laser scanning and unmanned aerial vehicle structure from motion to assess top of canopy structure in tropical forests. Interface Focus 8(2): 20170038. DOI: 10.1098/rsfs.2017.0038

Sankey T., Donager J., McVay J., Sankey J.B., 2017. UAV lidar and hyperspectral fusion for forest monitoring in the southwestern USA. Remote Sensing of Environment 195: 30-43. DOI: 10.1016/j.rse.2017.04.007

SAS, 2018. Base SAS 9.4 Procedures Guide: Statistical Procedures, Second Edition. Web: https://support.sas. com/documentation/cdl/en/procstat/66703/PDF/default/procstat.pdf. Accessed: 23.07.18

Singh P.S., Sharma M., Saikhom V., Chutia D., Gupta C., Chouhan A., Raju P.L.N., 2018. Towards generation of effective $3 \mathrm{D}$ surface models from UAV imagery using open source tools. Current Science 114(2): 9. DOI: $10.18520 / \mathrm{cs} / \mathrm{v} 114 / \mathrm{i} 02 / 314-321$
Snavely N., Seitz S.M., Szeliski R., 2008. Modeling the world from internet photo collections. International Journal of Computer Vision 80(2): 189-210. DOI: 10.1007/s11263-007-0107-3

Strîmbu V.F., Strîmbu B.M., 2015. A graph-based segmentation algorithm for tree crown extraction using airborne LiDAR data. ISPRS Journal of Photogrammetry and Remote Sensing 104: 30-43. DOI: 10.1016/j. isprsjprs.2015.01.018

Torresan C., Berton A., Carotenuto F., Di Gennaro S.F., Gioli B., Matese A., Miglietta F., Vagnoli C., Zaldei A., Wallace L., 2017. Forestry applications of UAVs in Europe: a review. International Journal of Remote Sensing 38(8-10): 2427-2447. DOI: 10.1080/01431161.2016.1252477

WallaceL., Lucieer A., Malenovský Z., TurnerD., Vopěnka P., 2016. Assessment of forest structure using two UAV Techniques: A comparison of airborne laser scanning and structure from motion (SfM) Point Clouds. Forests 7(3): 62. DOI: 10.3390/f7030062

Zahawi R.A., Dandois J.P., Holl K.D., Nadwodny D., Reid J.L., Ellis, E.C., 2015. Using lightweight unmanned aerial vehicles to monitor tropical forest recovery. Biological Conservation 186: 287-295. DOI: 10.1016/j. biocon.2015.03.031

Zhang J., Hu J., Lian J., Fan Z., Ouyang X., Ye W., 2016. Seeing the forest from drones: Testing the potential of lightweight drones as a tool for long-term forest monitoring. Biological Conservation 198: 60-69. DOI: 10.1016/j.biocon.2016.03.027 
